\title{
Planejamento e produção de um criostato de baixo custo para caracterização elétrica de materiais
}

(Planning and production of a low cost cryostat for electrical characterization of materials)

\author{
G.B. Torsoni ${ }^{\boxplus}$ e C.L. Carvalho \\ Grupo de Desenvolvimento e Aplicações de Materiais, Departamento de Física e Química, \\ Universidade Estadual Paulista "Julio de Mesquita Filho", Ilha Solteira, SP, Brasil \\ Recebido em 27/2/2012; Aceito em 24/5/2012; Publicado em 14/12/2012
}

\begin{abstract}
Os supercondutores a base de $\mathrm{BiSrCaCuO}$ apresentam três fases principais, $\mathrm{Bi}_{2} \mathrm{Sr}_{2} \mathrm{CuO}, \mathrm{Bi}_{2} \mathrm{Sr}_{2} \mathrm{CaCu}_{2} \mathrm{O}$ e $\mathrm{Bi}_{2} \mathrm{Sr}_{2} \mathrm{Ca}_{2} \mathrm{Cu}_{3} \mathrm{O}$ com temperaturas críticas de $20 \mathrm{~K}, 80 \mathrm{~K}$ e $110 \mathrm{~K}$, respectivamente. Desta forma, torna-se fundamental a caracterização elétrica de tais materiais a baixas temperaturas. Com este objetivo confeccionou-se um criostato para medidas de caracterização elétrica. Utilizando-se nitrogênio líquido (77 K), e controlando a pressão sobre o refrigerante, atingiu-se uma temperatura da ordem de $64 \mathrm{~K}$ (baseado em princípios termodinâmicos) abrangendo uma maior faixa de temperatura de medida, que permitiu identificar pelo menos duas fases supercondutoras no sistema BSCCO (2212 e 2223). Neste contexto, este sistema propicia uma relação custo/beneficio mais favorável, pois evita o uso de refrigerantes mais custosos como o hélio líquido. O criostato mostra experimentalmente a aplicação dos diagramas de fase PxT (Lei de Gay-Lussac) vistos nos cursos de termodinâmica, propiciando demonstrações experimentais que raras vezes são vistas em sala de aula.
\end{abstract}

Palavras-chave: caracterização criostato, supercondutor, princípios termodinâmicos, diagramas de fases.

Superconductor systems based on $\mathrm{BSCCO}(\mathrm{BiSrCaCuO})$ show three main structures, $\mathrm{Bi}_{2} \mathrm{Sr}_{2} \mathrm{CuO}_{2} \mathrm{Bi}_{2} \mathrm{Sr}_{2} \mathrm{Ca}_{2}$ $\mathrm{Cu}_{2} \mathrm{O}$, and $\mathrm{Bi}_{2} \mathrm{Sr}_{2} \mathrm{CaCu}_{3} \mathrm{O}$, with critical temperatures about $20 \mathrm{~K}, 80 \mathrm{~K}$, and $110 \mathrm{~K}$, respectively. Therefore, it is fundamental to characterize these systems at low temperatures. In this work it was projected a cryogenic system with capacity to reach temperatures below the liquid nitrogen temperature $(77 \mathrm{~K})$. Controlling the pressure on the liquid nitrogen system, according to usual thermodynamic principles, it has been possible to reach a range of temperatures above $64 \mathrm{~K}$ in the sample holder. With the availability of a larger temperature range, it became possible to identify at least two superconducting phases in the BSCCO system. There is a favorable cost/benefit ratio with respect to using liquid helium as the refrigerator system. Also, this cryostat provides an application of the standard p-T diagrams (Gay-Lussac law), which are studied in undergraduate courses, and can be used as a pedagogical experimental demonstration.

Keywords: characterization, cryostat, superconductor, thermodynamic principles phase diagrams.

\section{Introdução}

A rápida evolução da tecnologia pode ser observada através de exemplos comuns, tais como, celulares, televisores e microcomputadores cada vez menores. Essa evolução implica na necessidade de se obter dispositivos cada vez mais reduzidos e que tenha capacidade de conduzir sinais elétricos melhor do que os metais convencionais. Assim, nessa exigência se encaixam os materiais supercondutores de alta temperatura critica. Dentro dessa classe de supercondutores, comumente chamados de óxidos supercondutores, tem-se vários sistemas, tais como, os sistemas $\mathrm{LaBaCuO}, \mathrm{HgBaCaCuO}$ e $\mathrm{BiSrCaCuO}$, sendo que o último sistema pode apresentar 3 fases principais, ou seja, $\mathrm{Bi}_{2} \mathrm{Sr}_{2} \mathrm{CuO}$ (2201), $\mathrm{Bi}_{2} \mathrm{Sr}_{2} \mathrm{CaCu}_{2} \mathrm{O}$ (2212) e $\mathrm{Bi}_{2} \mathrm{Sr}_{2} \mathrm{Ca}_{2} \mathrm{Cu}_{3} \mathrm{O}$ (2223) com

\footnotetext{
${ }^{1}$ E-mail: gbtorsoni@yahoo.com.br.

Copyright by the Sociedade Brasileira de Física. Printed in Brazil.
}

as temperaturas críticas em torno de $3 \mathrm{~K}, 80 \mathrm{~K}$ e $110 \mathrm{~K}$, respectivamente $[1,2]$ Surgiu assim à necessidade de se caracterizar fases supercondutoras que estão abaixo da temperatura de vaporização do nitrogênio liquido $(77 \mathrm{~K})$, sendo que isso pode ser feito simplesmente baseando-se em princípios termodinâmicos normalmente vistos nas disciplinas de Termodinâmica e Física Estatística. Desta forma, sabe-se que existem quatro propriedades inter-relacionadas que podem ser usadas para descrever o estado de um gás: pressão $(P)$, volume $(V)$, temperatura $(T)$ e o número de moléculas ou mols $(n)$. Considerando-se e analisando-se as três leis dos gases [3-5];

\begin{tabular}{lll} 
Lei de Boyle & Lei de Charles & Lei de Avogadro \\
\hline$V \propto \frac{1}{P}$ & $V \propto T$ & $V \propto n$ \\
$(T, n$ constante $)$ & $(P, n$ constante $)$ & $(T, P$ constante $)$
\end{tabular}


as três leis podem ser combinadas e, tem-se como resultado que

$$
V \propto \frac{n T}{P} \quad \text { ou } \quad V=R\left(\frac{n T}{P}\right) .
$$

A constante de proporcionalidade, $R$, é a constante universal dos gases perfeitos, desde que sejam consideradas pressão e temperaturas longe dos estados de liquefação ou sublimação. A constante $R$ apresenta valor de $8.314 \mathrm{~J} / \mathrm{mol}$.K no sistema internacional de unidades. Reescrevendo a Eq (1) obtém-se a Eq. (2) [3-5]:

$$
P V=n R T \text {. }
$$

Como se pode notar, existe uma dependência linear da pressão com a temperatura, quando a pressão de um sistema fechado diminui, a temperatura também diminui e vice-versa, conforme mostrado na Eq. (2) Com o intuito de se trabalhar em temperaturas mais baixas que a do nitrogênio líquido (NL), confeccionou-se um criostato utilizando NL para o arrefecimento, onde foram estudadas as temperaturas de liquefação e solidificação do mesmo, como mostra o diagrama de fases do nitrogênio na Fig. 1.

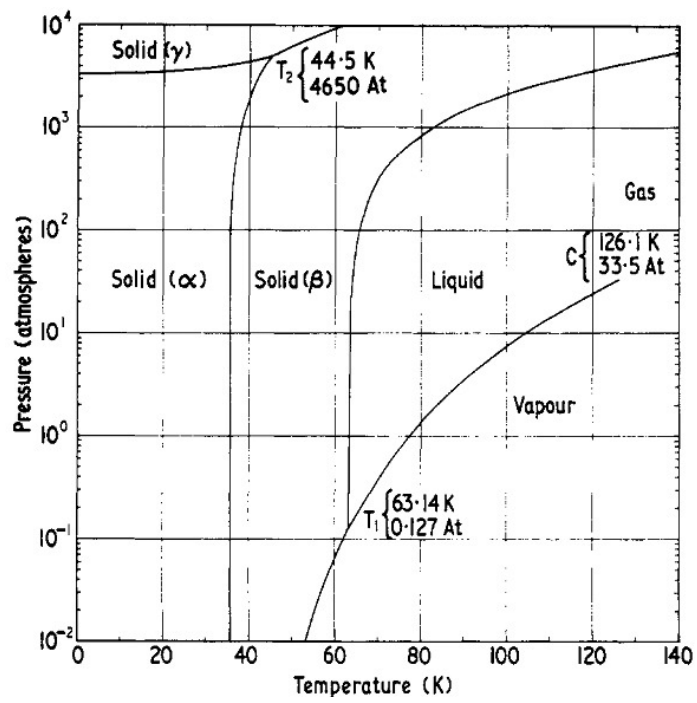

Figura 1 - Diagrama de fases do Nitrogênio [6].

É importante tomar o cuidado na escolha do refrigerante utilizado, pois alguns materiais apresentam um comportamento anômalo, ocorrendo a expansão térmica na solidificação, como por exemplo a água, no caso do nitrogênio segue a Lei de Charles, lembrando que $\mathrm{d} p / \mathrm{d} T$ é positivo [4,6] O estudo da Lei dos gases ideais é de grande importância, pois seu estudo é obrigatório desde o segundo grau até o ciclo básico de alguns cursos do nível superior (física, química, engenharia, etc). Do ponto de vista teórico, os princípios termodinâmicos, diagramas de fases e transformações isocóricas, são ensinados de forma satisfatória em sala de aula, no entanto, as demonstrações experimentais raras vezes são vistas. Portanto, neste trabalho, a cons- trução de um criostato de baixa temperatura (menores do que a de NL) teve o propósito de apresentar de maneira alternativa a demonstração da aplicação de algumas leis dos gases ideais e conceitos termodinâmicos, utilizando materiais facilmente encontrados no mercado nacional e de custo relativamente baixo. Esse equipamento pode ainda demonstrar para os estudantes como trabalhar de modo criterioso, pois quando fazemos uma medida o resultado não é obtido de forma tão direta, pois a medida está sujeita a erros, o que faz parte do cotidiano de um físico profissional. Outro ponto interessante para o ensino de física é a confecção de um manômetro muito simples a partir de um tubo em U preenchido com mercúrio (chamado líquido manométrico) cuja densidade é 13,6 vezes maior do que a da água. Um dos ramos do tubo está aberto à atmosfera e o outro está conectado com o criostato. A pressão no criostato, nesse tipo de barômetro, pode ser inferida pela altura $h$ do mercúrio $(\mathrm{Hg})$ como mostra a Eq. (3) [3]

$$
P=\rho_{l} g h,
$$

onde $P$ é a pressão registrada no manômetro, $\rho_{l}$ é a densidade do líquido manométrico, $g$ a gravidade e $h$ é a diferença de altura que atinge o líquido manométrico nos dois ramos.

\section{Materiais e métodos}

Na confecção do criostato utilizou-se aço inox a fim de se evitar a oxidação do metal e a condução de calor, pois a condutividade térmica do aço inox é $13,8 \mathrm{~W} / \mathrm{m}$.K [7], pequena quando comparada com o alumínio (205 $\mathrm{kW} / \mathrm{m} . \mathrm{K})$ ou o cobre $(385 \mathrm{~kW} / \mathrm{m} . \mathrm{K})$ [8], assim, com uma menor condutividade térmica o sistema fica melhor isolado para trocas de calor entre o meio externo e o interno. O mesmo foi projetado em duas partes, uma para isolação térmica (onde é feito o vácuo) e outra parte para o resfriamento (onde se encontra o nitrogênio líquido) como mostra a Fig. 2.

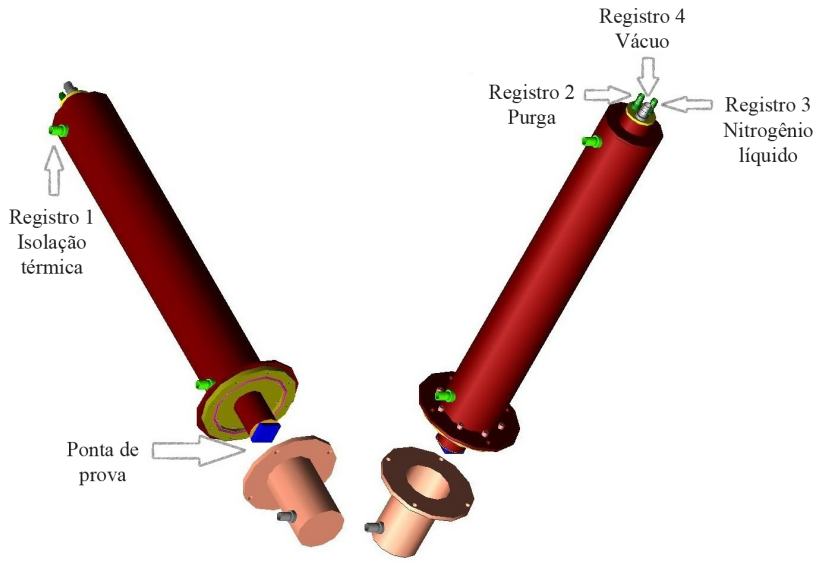

Figura 2 - Esquema do criostato mostrado em dois diferentes ângulos de visão. 
O registro 1 (ver Fig. 3) é utilizado para a realização do vácuo no compartimento de isolação térmica do recipiente contendo o refrigerante e a ponta de prova com relação o ambiente externo (parte 1), evitando assim condução de calor do ambiente para dentro do sistema refrigerado. $\mathrm{O}$ registro 2 tem a função de purgar o compartimento que contem o refrigerante, o abastecimento de refrigerante para dentro do respectivo compartimento é feito através do registro 3 já o registro 4 é utilizado para fazer o controle da pressão sobre o nitrogênio líquido. Na ponta de prova encontrase a amostra e o termopar do tipo $\mathrm{K}$, essa ponta de prova foi feita com material de cobre $(385 \mathrm{~kW} / \mathrm{m} . \mathrm{K})$ [8], para incrementar a condução de calor entre a amostra e o nitrogênio. O procedimento de funcionamento do criostato inicia-se com o abaixamento da temperatura, inserindo-se o nitrogênio líquido pelo registro 3 (parte 2) assim quando a temperatura do sistema estabilizar em torno de $77 \mathrm{~K}$, é cessado a inserção de nitrogênio liquido. Posteriormente inicia-se a aplicação de vácuo controlado, por um barômetro de precisão $( \pm 0,5 \mathrm{~mm}$ $\mathrm{Hg})$ no sistema contendo o refrigerante para assim baixar a temperatura do mesmo, de tal forma que a temperatura vá diminuindo gradativamente até em torno de $64 \mathrm{~K}$

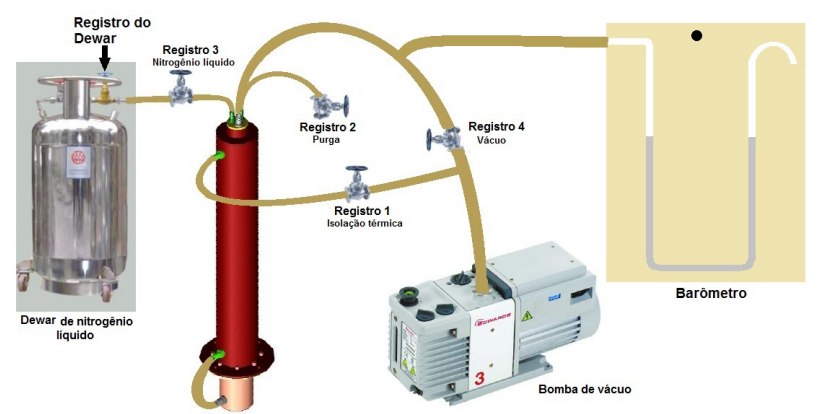

Figura 3 - Esquema de conexões e controle da pressão no sistema criogênico.

\section{Resultados e discussão}

Para o teste de caracterização do sistema criogênico, foi utilizado uma amostra de material supercondutor $\mathrm{BiPbSrCaCuO}$ com a estequiometria 2223, essa amostra foi feita a partir de processos químicos devido à facilidade em se efetuar a mistura e a homogeneização dos compostos precursores, isso quando comparado aos processos convencionais ou seja, mistura de óxidos. Assim, acetato de bismuto, cálcio, cobre, nitrato de chumbo e de estrôncio foram dissolvidos numa solução de ácido acético com concentração ácida controlada pelo acréscimo de hidróxido de amônio segundo a estequiometria citada. Com a adição de ácido oxálico a solução formou um precipitado contendo os respectivos íons metálicos. O pó foi seco numa estufa à $100{ }^{\circ} \mathrm{C}$ durante 24 horas. Após a secagem, o pó foi submetido a várias calcinações à uma temperatura de $600{ }^{\circ} \mathrm{C} / 24$ horas intercalado por triturações num almofariz de ágata. Uma quantidade de $6.0 \mathrm{~g}$ do pó obtido acima foi usado na fabricação de algumas pastilhas, esse pó foi prensado uniaxialmente à frio $\left(25^{\circ} \mathrm{C}\right)$ aplicando-se 9 toneladas, produzindo pastilhas com $20 \mathrm{~mm}$ de diâmetro e $1.0 \mathrm{~mm}$ de espessura. As pastilhas foram sinterizadas à $840{ }^{\circ} \mathrm{C}$ durante 100 horas, num forno EDG-1700, em atmosfera ambiente. O tratamento térmico à temperatura de 840 ${ }^{\circ} \mathrm{C}$ foi aplicado devido a estudos desenvolvidos anteriormente com esse mesmo tipo de material. Uma taxa de $120{ }^{\circ} \mathrm{C} / \mathrm{h}$ foi usada no aquecimento e $300{ }^{\circ} \mathrm{C} / \mathrm{h}$ para o resfriamento [9] Uma amostra do material citado acima foi colocada no criostato, onde foi efetuada uma medida elétrica da resistência elétrica em função da temperatura usando o método das 4 pontas DC, afim de se estabelecer qual a temperatura crítica, Tc do material, onde foi obtido o gráfico mostrado na Fig 4.

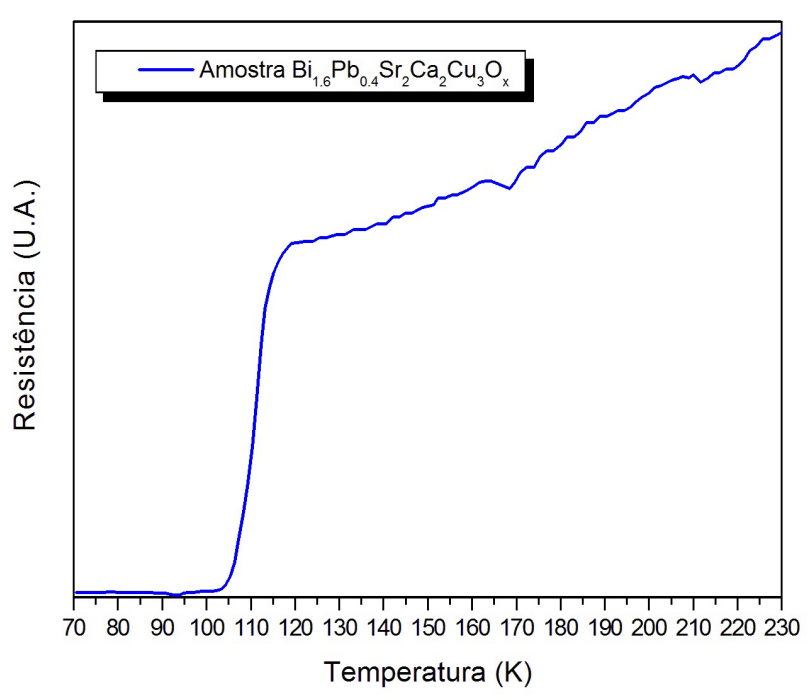

Figura 4 - Gráfico da resistência normalizada em função da temperatura para a amostra de $\mathrm{Bi}_{1.6} \mathrm{~Pb}_{0.4} \mathrm{Sr}_{2} \mathrm{Ca}_{2} \mathrm{Cu}_{3} \mathrm{O}_{x}$.

A medida mostra o comportamento perfeito da transição supercondutora em torno de $110 \mathrm{~K}$, temperatura característica da fase de 2223, confirmando assim a confiabilidade e a eficiência do equipamento. Usando nitrogênio líquido para baixar a temperatura até $78 \mathrm{~K}$, depois com a aplicação do vácuo, esta medida foi feita até $70 \mathrm{~K}$, obtendo-se assim uma faixa maior para esse tipo de medida.

\section{Conclusões}

Usando nitrogênio líquido (77 K), e aplicação de vácuo controlado sobre o sistema contendo o refrigerante, chegou-se a uma temperatura de $64 \mathrm{~K}$ abrangendo uma gama maior de medição. Neste contexto, este sistema oferece uma relação custo / benefício mais favorável, evitando o uso de refrigerantes mais caros, como é o caso do hélio líquido. Ressaltando que, experimento 
semelhante foi efetuado em outro sistema e o resultado obtido da temperatura de transição supercondutora foi idêntico ao apresentado pelo criostato desenvolvido neste trabalho [10] Com a produção e apresentação do criostato para estudantes do Ensino Médio e superior mostra de maneira clara, que as teorias que regem a termodinâmica são eficazes mesmo quando se trata de um sistema não ideal, vários equipamentos utilizados no dia-a-dia podem ser explicados a partir destes conceitos básicos termodinâmicos, como por exemplo refrigerador, motor do carro e garrafa térmica

\section{Agradecimentos}

Os autores são gratos à CAPES, FAPESP, CNPq e a Oficina Mecânica do DFQ pelo suporte financeiro e técnico, respectivamente.

\section{Referências}

[1] H.J. Lim and J.G. Byrne, Metallurgical and Materials Transactions B 28, 425 (1997).
[2] J.R Schrieffer, Handbook of High-Temperature Superconductivity Theory and Experiment (Springer, New York, 2007), p. 627.

[3] D. Halliday, R. Resnick and J. Walker, Fundamentals of Physics (Wiley, New York, 2003), v. 6, p. 1328.

[4] F.W. Sears and G.L. Salinger, Thermodynamics, Kinetic Theory, and Statistical Thermodynamics (AddisonWesley, New York, 1975), p. 454

[5] H.B. Callen, Thermodynamics and An Introduction to Thermostatistics (Wiley New York, 1985), p. 493.

[6] E.M. Wray, Physics Education 7, 243 (1972)

[7] J.G. Hust and L.L. Sparks, Thermal Conductivity of Austenitic Stainless Steel, SRM 735, from 5 to $280 \mathrm{~K}$ (Supt. of Docs., U.S. Govt. Print. Off., U.S. National Bureau of Standards 1972), p. 15

[8] F.W. Sears and M.W. Zemansky, University Physics (Addison-Wesley, New York, 1970), p. 1632

[9] C L. Carvalho, Preparação e Caracterização de Supercondutores BSCCO em Forma de Filmes e Cerâmicas Obtidos por Precursores Poliméricos Tese de Doutorado Universidade Estadual Paulista, 1999.

[10] G.B. Torsoni, in: Anais do 170 CBECIMat, Campos do Jordão, 2006, p. 512 